\title{
Análise crítica da rotulagem e medidas caseiras de margarinas, cremes vegetais, manteigas, requeijões e azeites de dendê
}

\author{
Critical analysis of labeling and household \\ measures of margarine, vegetable cream, butter, \\ cream cheese, and palm oil
}

\section{Abstract}

SOUZA, A. A.; ALMEIDA, D. T.; BARROS, H. D.; MACHADO, E. R.; PUMAR, M. Critical analysis of labeling and household measures of margarine, vegetable cream, butter, cream cheese, and palm oil. Nutrire: rev. Soc. Bras. Alim. Nutr. = J. Brazilian Soc. Food Nutr., São Paulo, SP, v. 39, n. 1, p. 1-16, abr. 2014.

The aim of this study was to determine whether nutrition labeling of foods containing lipid is consistent with the Brazilian Legislation, as well as whether the portions described on the label match the volumetric capacity of tablespoons. We analyzed the labels of 23 packages of margarine, 11 packages of vegetable cream, 45 packages of butter, 41 jars of cream cheese, and 12 bottles of palm oil, identified through online search and/or commercially available. A sample of each food group was weighed in 30 different types of level tablespoons. We verified that 20.6; 79.4 and $17.4 \%$ of the margarine and vegetables cream analyzed were designated as "bydrogenated", "interesterified", and "trans-fat", respectively; 11.8\% were designated as "butter + margarine", which is not in accordance with the legislation. There was heterogeneity in the levels of sodium declared, with mean values ranging from $50.0-94.2 \mathrm{mg} / 10 \mathrm{~g}$ for margarine and vegetable cream, $1.0-115.0 \mathrm{mg} / 10 \mathrm{~g}$ for butter, and 106.3-174.3 mg/30 g for cream cheese. The average cholesterol content declared in butter ranged from 35.0 to $196.0 \mathrm{mg} / 10 \mathrm{~g}$. The labeling of palm oil showed no list of ingredients in 50\% of the products assessed and incorrect statement of saturated fat content was present in $41.6 \%$ of the samples. The difference between the tablespoons ranged from 232.0 to $255.0 \%$. It is worth noting that only 6.7; 6. 7; 5.0 and 4.4\% presented the volumetric capacity of the tablespoons for margarine and vegetable cream, butter, cream cheese, and palm oil, respectively, in accordance with the legislation. The results demonstrate the need for standardization of tablespoons, updating, overt and efficient monitoring regarding the labeling of lipid foods in Brazil.

Keywords: Labeling. Nutrition information. Portions. Household measures.
ANDRESSA ALVES DE SOUZA'; DEUSDELIA TEIXEIRA DE

ALMEIDA';

HILDA DUVAL BARROS ${ }^{3}$;

ELIANA RODRIGUES MACHADO4; MATILDE PUMAR ${ }^{3}$

${ }^{1}$ Graduanda do Curso de Nutrição, Universidade do Estado do Rio de Janeiro - UERJ.

2Professora Associada da Escola de Nutrição, Universidade Federal da Bahia - UFBA.

${ }^{3}$ Professora Adjunta do Instituto de Nutrição, Universidade do Estado do Rio de Janeiro - UERJ. ${ }^{4}$ Pesquisadora e Tecnologista em Saúde Pública, Instituto Nacional

de Controle de Qualidade em Saúde - INCQS, Fundação Oswaldo Cruz.

Endereço para correspondência: Universidade Federal da Bahia - UFBA.

Escola de Nutrição.

Deusdelia Teixeira de Almeida. Rua Araújo Pinho, 32, Canela. CEP 40110-150.

Salvador - BA - Brasil. E-mail: deliata@uol.com.br Agradecimentos: À FAPESB (Termo No: BOL1784/2010), ao INCQS/ FIOCRUZ/RJ, pelo acolhimento, apoio financeiro e estrutura laboratorial; ao projeto IMVISA/ Instituto de Nutrição/UERJ, pela oportunidade de participação e pela concessão da bolsa da aluna Andressa Alves de Souza; e aos funcionários do INCQS, pelo empréstimo das diversas colheres de sopa para o uso neste experimento. 


\section{Resumen}

El objetivo de este estudio fue determinar si el etiquetado nutricional de los alimentos fuente de lípidos está de acuerdo con la ley brasileña vigente, asi como si las porciones descritas en la etiqueta coinciden con la capacidad volumétrica de cucharadas soperas. Se analizaron las etiquetas de 23 margarinas, 11 cremas vegetales, 45 mantequillas, 41 requesones y 12 aceites de palma, productos que fueron identificados a través de una búsqueda por internet y/o adquiridos en el comercio. Se pesó una muestra de cada alimento, usando 30 diferentes tipos de cucharas soperas. Se verificó que el 20,6; 79, 4 y $17,4 \%$ de las margarinas y cremas vegetales se etiquetaron como "bidrogenadas", "interesterificadas" y "con grasa trans", respectivamente; también se verificó que el 11,8\% se etiquetó como "mantequilla + margarina", item no previsto en la legislación. Se observó beterogeneidad en los niveles de sodio declarados, con los siguientes valores medios: 50,0-94,2 $\mathrm{mg} / 10 \mathrm{~g}$ en las margarinas y cremas vegetales; 1,0-115 mg/10 g en las mantequillas y 106,3$174,3 \mathrm{mg} / 30 \mathrm{~g}$ en los requesones. El contenido medio de colesterol declarado en las mantequillas osciló entre 35,0-196,0 mg/10g. El etiquetado del aceite de palma carecía del listado de ingredientes en el $50 \%$ de los productos, y el nivel de grasa saturada estaba etiquetado de forma inexacta en el 41,6\% de las muestras. La diferencia entre cucharadas soperas varió desde 232,0255,0\%, puesto que el 6, 7; 6, 7; 5, oy 4,4\% de las margarinas $y$ cremas vegetales, mantequillas, requesones y aceites de palma, respectivamente, presentaron la capacidad volumétrica establecida en la legislación. Los resultados demuestran la necesidad de estandarización de las cucharadas soperas, actualización, fiscalización rigurosa y eficiente en el etiquetado de los alimentos lipídicos en Brasil.

Palabras clave: Etiquetado. Información nutricional. Porciones. Medidas caseras.
O objetivo do presente estudo foi verificar se a rotulagem nutricional de alimentos fontes de lipidios está de acordo com a legislação brasileira vigente, bem como se as porções descritas no rótulo coincidem com a capacidade volumétrica de colheres de sopa. Foram analisados os rótulos de 23 margarinas, 11 cremes vegetais, 45 manteigas, 41 requeijões e 12 azeites de dendê, identificados por meio de busca on-line e/ou adquiridos no comércio. Pesou-se uma amostra de cada alimento em 30 diferentes tipos de colberes de sopa niveladas. Verificou-se que 20,6\%; 79,4\% e $17,4 \%$ das margarinas e cremes vegetais foram designadas como "bidrogenadas", "interesterificadas" e "com gordura trans", respectivamente; $11,8 \%$ foram denominadas "manteiga + margarina", item não previsto na legislação. Verificou-se heterogeneidade nos teores de sódio declarados. Os valores médios encontrados foram: 50,0-94,2 mg/10 g nas margarinas e cremes vegetais; $1,0-115,0 \mathrm{mg} / 10 \mathrm{~g}$ nas manteigas $e$ 106,3-174,3 mg/30 g, nos requeijões. O teor médio de colesterol declarado nas manteigas variou entre 35,0-196,0 mg/10g. A rotulagem do azeite de dendê demonstrou ausência da listagem de ingredientes em 50\% dos produtos e a declaração incorreta do teor de gordura saturada esteve presente em 41,6\% dessas amostras. A diferença entre as colberes variou entre 232,0\% e 255,0\%, sendo que 6, 7\%; 6, 7\%; 5,0\% e 4,4\% destas apresentaram capacidade volumétrica para margarinas e cremes vegetais, manteigas, requeijões $e$ azeites de dendê, respectivamente, em conformidade com a legislação. Os resultados demonstram a necessidade de uma padronização das colberes de sopa, atualização, fiscalização ostensiva e eficiente quanto à rotulagem de alimentos lipídicos no Brasil.

Palavras-chave: Rotulagem. Informação nutricional. Porções. Medidas caseiras. 


\section{INTRODUÇÃO}

A estreita relação entre doenças crônicas não transmissíveis e as práticas alimentares inadequadas, tais como redução do consumo de cereais, oleaginosas, leguminosas, frutas e vegetais, e o excessivo consumo de sal, gorduras saturadas, carboidratos simples e alimentos industrializados, levou a Organização Mundial de Saúde (OMS), em 2005, a promover a "Estratégia Global em Alimentação Saudável, Atividade Física e Saúde". Este documento busca a redução de fatores de risco de doenças não transmissíveis associadas a uma alimentação inadequada e ao sedentarismo, mediante uma ação de saúde pública essencial e medidas de promoção da saúde e prevenção da morbidade ${ }^{1,2}$.

O Brasil foi um dos países que se tornaram signatários dessa Estratégia Global; dentre suas ações, destaca-se a elaboração da agenda de promoção da alimentação saudável qualificada com a edição do Guia Alimentar para a População Brasileira ${ }^{3}$, instrumento oficial que define as diretrizes alimentares para serem utilizadas na orientação de escolhas mais saudáveis de alimentos e medidas legislativas que determinam padrões de identidade, qualidade e rotulagem de alimentos industrializados embalados.

As informações nutricionais, referentes ao produto industrializado embalado, devem ser apresentadas em porções e medidas caseiras correspondentes reguladas pela $\mathrm{RDC} \mathrm{n}{ }^{\circ} 359^{4}$, devem conter o teor e o percentual de valores diários para cada nutriente: carboidratos; proteínas; gorduras totais; gorduras saturadas; gorduras trans; fibra alimentar; sódio e valor energético, segundo a $\mathrm{RDC} \mathrm{n}^{\circ} 360^{5}$.

Converter porções em medidas caseiras correspondentes é uma prática que vem sendo adotada universalmente desde princípios da década de 1940, tornando-se imprescindível como ferramenta nas recomendações diárias de ingestão de nutrientes, em estudos sobre inquéritos alimentares, na elaboração de ficha de análise de preparação de cardápios e em receitas culinárias ${ }^{6}$.

A regulamentação de rotulagem no Brasil foi uma grande conquista para o consumidor, no entanto, estudos têm apontado falhas na legislação, principalmente no que tange à informação nutricional ${ }^{7,8}$. Além disto, se observa que, no que concerne às porções e medidas caseiras, o Brasil não dispõe de regulamentações de padrões de utensílios domésticos, os quais apresentam ampla variação de capacidade volumétrica, o que pode comprometer propósitos enunciados nesses documentos, principalmente os relacionados à ingestão de nutrientes ${ }^{6}$.

O presente trabalho teve como objetivo avaliar se as informações nutricionais, ingredientes, medidas caseiras e porções dos rótulos de margarinas e cremes vegetais, manteigas, requeijões e azeites de dendê estariam em conformidade com a legislação vigente e apontar sugestões para melhor adequação destes rótulos às necessidades do consumidor.

\section{METODOLOGIA}

\section{Produtos avaliados}

A identificação dos produtos foi realizada entre outubro e dezembro de 2011, por meio de busca on-line no buscador Google web e/ou imagem, utilizando como estratégia de identificação as palavras: margarinas, manteiga, cremes vegetais, azeite de dendê, requeijão, e/ou suas respectivas 
classificações, sendo as informações recopiladas apenas dos sites dos fabricantes. Dúvidas sobre rotulagem foram esclarecidas por meio de aquisição da informação em supermercados da cidade do Rio de Janeiro, Brasil e/ou serviço de atendimento ao cliente (SAC). Especificamente para o azeite de dendê, além dessas estratégias, os rótulos foram adquiridos em supermercados e feiras da cidade de Salvador-Bahia, num total de 12 diferentes marcas, enumeradas de 1 a 12.

Foram analisados 132 rótulos, sendo 23 de margarinas (6 margarinas com denominações diversas, 6 light, 4 cremosas, 3 extra cremosas, 4 designadas mix de margarina + manteiga); 11 de cremes vegetais; 45 de manteigas (19 de primeira qualidade, 10 extra com sal, 7 extra sem sal, 7 comuns e 2 sem classificação); 41 de requeijões (22 tradicionais, 14 light e 5 com gordura e amido) e 12 de azeites de dendê ( 2 com denominação de oleína, 3 misturas de dendê + soja e 7 integrais).

\section{INFORMAÇÕES NUTRICIONAIS, DESIGNAÇÃO E COMPOSIÇÃO DOS PRODUTOS}

A avaliação de rotulagem foi realizada por meio de uma ficha de avaliação padronizada, desenvolvida pelos pesquisadores do presente estudo, de acordo com todos os itens descritos na legislação brasileira, referentes a informação nutricional, ingredientes, porções e medidas caseiras. As legislações consultadas foram a Portaria n 146 - Regulamento Técnico de Identidade e Qualidade de Manteiga'; Portaria n 359 - Regulamento Técnico para Fixação de identidade e Qualidade de Requeijão ou Requesón ${ }^{10}$; Portaria n 372 - Regulamento Técnico para Fixação de Identidade e Qualidade de Margarina ${ }^{11}$; Resolução da Diretoria Colegiada (RDC) no 54 - Regulamento Técnico Referente à Informação Nutricional Complementar ${ }^{12}$; Resolução $n^{\circ} 4$-institui o produto denominado Manteiga Comum ${ }^{13}$; RDC n³59 - Regulamento Técnico de Porções de Alimentos Embalados para Fins de Rotulagem Nutricional $^{4}$, que aborda porção e medida caseira; RDC n³60 - Regulamento Técnico sobre Rotulagem Nutricional de Alimentos Embalados ${ }^{5}$; RDC $\mathrm{n}^{\circ} 270$, Regulamento Técnico para Óleos Vegetais, Gorduras Vegetais e Cremes Vegetais ${ }^{14}$.

\section{VERIFICAÇÃo dA PORÇÃo E MEdIDA CASEIRA CORRESPONDENTE}

Com a finalidade de verificar porção e medida caseira correspondentes, declaradas nos rótulos, aos alimentos analisados, foram empregadas 30 diferentes colheres de sopa, cedidas como empréstimo por funcionários da Fiocruz-Rio e numericamente identificadas. Os alimentos foram mensurados no Laboratório de óleos e gorduras do Instituto Nacional de Qualidade em Saúde (INCQS), em novembro de 2011, empregando-se uma balança semianalítica (RADWAG modelo PS750/C/2). O azeite de dendê (comercializado como oleína de palma) e a manteiga (de primeira qualidade) foram pesados em triplicata e o requeijão tradicional, margarina cremosa em duplicata, em temperatura ambiente, de acordo com a técnica de PHILIPPI ${ }^{15}$, estabelecendo-se como medida para todas as colheres e para todos os alimentos a colher de sopa nivelada como padronização para mensuração e posterior comparação analítica.

\section{AnÁlise estatística}

Inicialmente os dados foram copilados para o programa Excel e verificadas as informações sobre a porção; medida caseira; dieta baseada em $2000 \mathrm{kcal}$; tabela nutricional e ingredientes. Depois de revisados, os dados foram transferidos para o SPSS (Statistical Product and Service Solutions), versão 13.0 e as variáveis analisadas por meio de frequência e análises descritivas. O teste de Kruskal- 
Wallis foi empregado para comparação dos itens analisados na rotulagem entre os diversos tipos de alimentos (Tabelas 1, 2, 3), exceto para o azeite de dendê, cujos dados de rotulagem de todas as marcas estão apresentados na Tabela 4. A significância estatística foi considerada, admitindo-se um nível crítico de $\mathrm{p} \leq 0,05$.

Tabela 1 - Informações nutricionais, de rótulos de margarinas e cremes vegetais obtidos por meio de busca on-line, 2011

\begin{tabular}{|c|c|c|c|c|c|c|}
\hline Classificação & $\begin{array}{l}\text { Informações } \\
\text { nutricionais por } \\
\text { porção (10 g) }\end{array}$ & $\mathbf{n}$ & Mínimo & Máximo & Média & $\begin{array}{l}\text { Desvio } \\
\text { padrão }\end{array}$ \\
\hline Margarinas & \multirow{6}{*}{ Valor Energético (kcal) } & 6 & 36,0 & 72,0 & $52,5^{\mathrm{a}}$ & 12,4 \\
\hline Margarina light & & 6 & 27,0 & 34,0 & $31,5^{\mathrm{b}}$ & 2,4 \\
\hline Margarina cremosa & & 4 & 45,0 & 72,0 & $57,5^{\mathrm{cd}}$ & 14,5 \\
\hline Marg. extra cremosa & & 3 & 54,0 & 74,0 & $62,3^{\mathrm{cd}}$ & 10,4 \\
\hline Margarina + Manteiga & & 4 & 45,0 & 74,0 & $66,8^{\text {ecd }}$ & 14,5 \\
\hline Creme vegetal & & 11 & 18,0 & 59,0 & $31,9^{f}$ & 12,1 \\
\hline Margarinas & \multirow{6}{*}{ Gorduras Totais (g) } & 6 & 4,0 & 8,0 & $5,8^{\mathrm{a}}$ & 1,4 \\
\hline Margarina light & & 6 & 3,0 & 3,8 & $3,5^{\mathrm{b}}$ & 0,3 \\
\hline Margarina cremosa & & 4 & 5,0 & 8,0 & $6,4^{\mathrm{cd}}$ & 1,6 \\
\hline Marg. extra cremosa & & 3 & 6,0 & 8,2 & $6,9^{\mathrm{cd}}$ & 1,2 \\
\hline Margarina + Manteiga & & 4 & 5,0 & 8,2 & $7,4^{\text {ecd }}$ & 1,6 \\
\hline Creme vegetal & & 11 & 2,0 & 6,5 & $3,6^{\mathrm{f}}$ & 1,3 \\
\hline Margarinas & \multirow{6}{*}{ Gorduras Saturadas (g) } & 6 & 1,4 & 2,2 & $1,7^{\mathrm{a}}$ & 0,3 \\
\hline Margarina light & & 6 & 0,8 & 1,2 & $1,1^{\mathrm{b}}$ & 0,2 \\
\hline Margarina cremosa & & 4 & 1,7 & 2,4 & $2,0^{\mathrm{cd}}$ & 0,4 \\
\hline Marg. extra cremosa & & 3 & 1,1 & 2,3 & $1,9^{\mathrm{cd}}$ & 0,7 \\
\hline Margarina + Manteiga & & 4 & 1,7 & 3,6 & $2,9^{\text {ecd }}$ & 0,9 \\
\hline Creme vegetal & & 11 & 0,4 & 2,1 & $0,9^{f}$ & 0,5 \\
\hline Margarinas & \multirow{6}{*}{ Sódio (mg) } & 6 & 78,0 & 119,0 & $94,2^{\mathrm{a}}$ & 19,4 \\
\hline Margarina light & & 6 & 0,0 & 112,0 & $73,0^{\mathrm{b}}$ & 39,1 \\
\hline Margarina cremosa & & 4 & 0,0 & 104,0 & $56,5^{\mathrm{cd}}$ & 42,8 \\
\hline Marg. extra cremosa & & 3 & 52,0 & 98,0 & $76,0^{\mathrm{cd}}$ & 23,1 \\
\hline Margarina + Manteiga & & 4 & 0,0 & 104,0 & $50,0^{\text {ecd }}$ & 42,5 \\
\hline Creme vegetal & & 11 & 35,0 & 102,0 & $74,6^{\mathrm{f}}$ & 20,5 \\
\hline
\end{tabular}

n: número de rótulos; letras diferentes, na mesma coluna, indicam diferenças significativas entre os tratamentos, teste KruskalWallis $(\mathrm{p}<0,05)$. 
Tabela 2 - Informações nutricionais de rótulos de manteigas obtidos por meio de busca on-line, 2011

\begin{tabular}{|c|c|c|c|c|c|c|}
\hline Classificação & $\begin{array}{l}\text { Informações nutricionais } \\
\text { por porção }(10 \mathrm{~g})\end{array}$ & $\mathbf{n}$ & Mínimo & Máximo & Média & $\begin{array}{l}\text { Desvio } \\
\text { padrão }\end{array}$ \\
\hline Primeira qualidade & \multirow{5}{*}{ Valor Energético (kcal) } & 19 & 71,0 & 117,0 & $83,6^{\mathrm{a}}$ & 16,4 \\
\hline Extra com sal & & 10 & 73,0 & 230,0 & $91,1^{\mathrm{a}}$ & 48,8 \\
\hline Extra sem sal & & 7 & 73,0 & 230,0 & $99,3^{\mathrm{a}}$ & 57,9 \\
\hline Comum & & 7 & 56,0 & 129,0 & $83,0^{\mathrm{a}}$ & 24,1 \\
\hline Sem Classificação & & 2 & 74,0 & 100,0 & $87,0^{\mathrm{a}}$ & 18,4 \\
\hline Primeira qualidade & \multirow{5}{*}{ Gorduras Totais (g) } & 19 & 6,2 & 11,4 & $8,6^{\mathrm{a}}$ & 1,3 \\
\hline Extra com sal & & 10 & 8,0 & 25,0 & $10,0^{\mathrm{a}}$ & 5,3 \\
\hline Extra sem sal & & 7 & 8,1 & 25,0 & $10,9^{\mathrm{a}}$ & 6,2 \\
\hline Comum & & 7 & 8,0 & 12,0 & $8,9^{\mathrm{a}}$ & 1,4 \\
\hline Sem Classificação & & 2 & 8,3 & 14,0 & $11,2^{\mathrm{a}}$ & 4,0 \\
\hline Primeira qualidade & \multirow{5}{*}{ Gorduras Saturadas (g) } & 19 & 3,7 & 8,0 & $5,7^{\mathrm{a}}$ & 1,1 \\
\hline Extra com sal & & 10 & 4,6 & 17,0 & $6,4^{\mathrm{a}}$ & 3,8 \\
\hline Extra sem sal & & 7 & 4,6 & 17,0 & $6,9^{\mathrm{a}}$ & 4,5 \\
\hline Comum & & 7 & 4,0 & 7,0 & $5,4^{\mathrm{a}}$ & 0,9 \\
\hline Sem Classificação & & 2 & 4,8 & 7,0 & $5,9^{\mathrm{a}}$ & 1,6 \\
\hline Primeira qualidade & \multirow{5}{*}{ Gorduras Trans (g) } & 19 & 0,0 & 90,0 & $14,1^{\mathrm{a}}$ & 33,2 \\
\hline Extra com sal & & 10 & 0,0 & 88,0 & $9,1^{\mathrm{a}}$ & 27,7 \\
\hline Extra sem sal & & 7 & 0,0 & 88,0 & $12,9^{\mathrm{a}}$ & 33,1 \\
\hline Comum & & 7 & 0,0 & 88,0 & $12,6^{\mathrm{a}}$ & 33,2 \\
\hline Sem Classificação & & 2 & 0,0 & 90,0 & $45,0^{\mathrm{a}}$ & 63,6 \\
\hline Primeira qualidade & \multirow{5}{*}{ Sódio (mg) } & 19 & 10,0 & 150,0 & $80,9^{\mathrm{a}}$ & 33,5 \\
\hline Extra com sal & & 10 & 0,0 & 120,0 & $61,5^{\mathrm{ab}}$ & 34,8 \\
\hline Extra sem sal & & 6 & 0,0 & 6,0 & $1,0^{c}$ & 2,4 \\
\hline Comum & & 7 & 0,0 & 95,0 & $54,4^{\mathrm{de}}$ & 33,3 \\
\hline Sem Classificação & & 2 & 90,0 & 140,0 & $115,0^{\text {de }}$ & 35,4 \\
\hline
\end{tabular}

n:número de rótulos; letras diferentes, na mesma coluna, indicam diferenças significativas entre os tratamentos, teste KruskalWallis $(\mathrm{p}<0,05)$.

\section{RESULTADOS E DISCUSSÃO}

\section{INFORMAÇÕES NUTRICIONAIS, DESIGNAÇÃO E COMPOSIÇÃO DOS PRODUTOS}

\section{MARGARINAS E CREMES VEGETAIS}

De acordo com a legislação brasileira ${ }^{11}$, a margarina é o produto gorduroso em emulsão estável, com leite ou seus constituintes ou derivados, com no máximo $95 \%$ de lipídios totais, sendo classificadas de acordo com este teor, cujo valor deve estar declarado no painel principal do rótulo de forma clara, destacada e precisa. A RDC no $270^{14}$ define os Cremes Vegetais como "o produto 
Tabela 3 - Informações nutricionais de rótulos de requeijões obtidos por meio de busca on-line, 2011

\begin{tabular}{|c|c|c|c|c|c|c|}
\hline Classificação & $\begin{array}{l}\text { Informações nutricionais } \\
\text { por porção }(30 \mathrm{~g})\end{array}$ & n & Mínimo & Máximo & Média & $\begin{array}{l}\text { Desvio } \\
\text { padrão }\end{array}$ \\
\hline Tradicional & \multirow{3}{*}{ Valor Energético (kcal) } & 22 & 71,0 & 136,0 & $87,7^{\mathrm{a}}$ & 16,2 \\
\hline Light & & 14 & 41,0 & 83,0 & $57,4^{\mathrm{b}}$ & 12,0 \\
\hline Com gordura e amido & & 5 & 60,0 & 82,0 & $70,2^{c}$ & 9,1 \\
\hline Tradicional & \multirow{3}{*}{ Carboidratos (g) } & 22 & 0,0 & 2,0 & $0,7^{\mathrm{a}}$ & 0,6 \\
\hline Light & & 14 & 0,0 & 2,0 & $0,9^{\mathrm{b}}$ & 0,6 \\
\hline Com gordura e amido & & 5 & 1,0 & 3,0 & $1,8^{\mathrm{c}}$ & 0,8 \\
\hline Tradicional & \multirow{3}{*}{ Proteínas (g) } & 22 & 2 & 6,7 & $3,2^{\mathrm{a}}$ & 1,1 \\
\hline Light & & 14 & 3,1 & 6,5 & $4,1^{\mathrm{b}}$ & 1,0 \\
\hline Com gordura e amido & & 5 & 1,1 & 6 & $2,8^{\mathrm{c}}$ & 2,0 \\
\hline Tradicional & \multirow{3}{*}{ Gorduras Totais (g) } & 22 & 6,1 & 12,3 & $7,9^{\mathrm{a}}$ & 1,9 \\
\hline Light & & 14 & 2,7 & 7,6 & $4,1^{\mathrm{b}}$ & 1,3 \\
\hline Com gordura e amido & & 5 & 5,2 & 6,3 & $5,8^{\mathrm{c}}$ & 0,5 \\
\hline Tradicional & \multirow{3}{*}{ Gorduras Saturadas (g) } & 21 & 3,6 & 8,6 & $5,0^{\mathrm{a}}$ & 1,3 \\
\hline Light & & 13 & 1,7 & 4,5 & $2,6^{\mathrm{b}}$ & 0,8 \\
\hline Com gordura e amido & & 5 & 2,2 & 4,7 & $3,7^{c}$ & 0,9 \\
\hline Tradicional & \multirow{3}{*}{ Sódio (mg) } & 22 & 80,0 & 330,0 & $174,3^{\mathrm{a}}$ & 74,3 \\
\hline Light & & 14 & 44,9 & 407,0 & $191,3^{\mathrm{a}}$ & 91,7 \\
\hline Com gordura e amido & & 5 & 0,22 & 164,3 & $106,3^{\mathrm{b}}$ & 62,6 \\
\hline Tradicional & \multirow{2}{*}{ Cálcio (mg) } & 10 & 4 & 170 & $75,0^{\mathrm{a}}$ & 60,2 \\
\hline Light & & 7 & 8 & 170 & $89,9^{\mathrm{a}}$ & 65,2 \\
\hline
\end{tabular}

n: número de rótulos; letras diferentes, na mesma coluna, indicam diferenças significativas entre os tratamentos, teste Kruskal-Wallis $(\mathrm{p}<0,05)$.

em forma de emulsão plástica ou fluida, constituído principalmente de água e óleo vegetal e ou gordura vegetal, podendo ser adicionado de outro(s) ingrediente(s)".

A análise da rotulagem das margarinas e cremes vegetais demonstrou que estes continham a especificação da marca, seguida de outras nomenclaturas, tais como: "ao leite"; "sabor de milho"; "50\% lipidios"; "vitaminada"; "família"; "cremes vegetais sabor manteiga", sugerindo a ideia de opções mais saudáveis e saborosas. Isto é decorrência de leis que permitem que os fabricantes denominem os produtos das mais variadas formas, dificultando o entendimento dos rótulos por parte dos consumidores.

Observou-se que os produtos apresentaram conformidade quanto aos ingredientes e aditivos permitidos ${ }^{11}$. Ressalta-se, contudo, que duas marcas de cremes vegetais declaravam gordura animal 


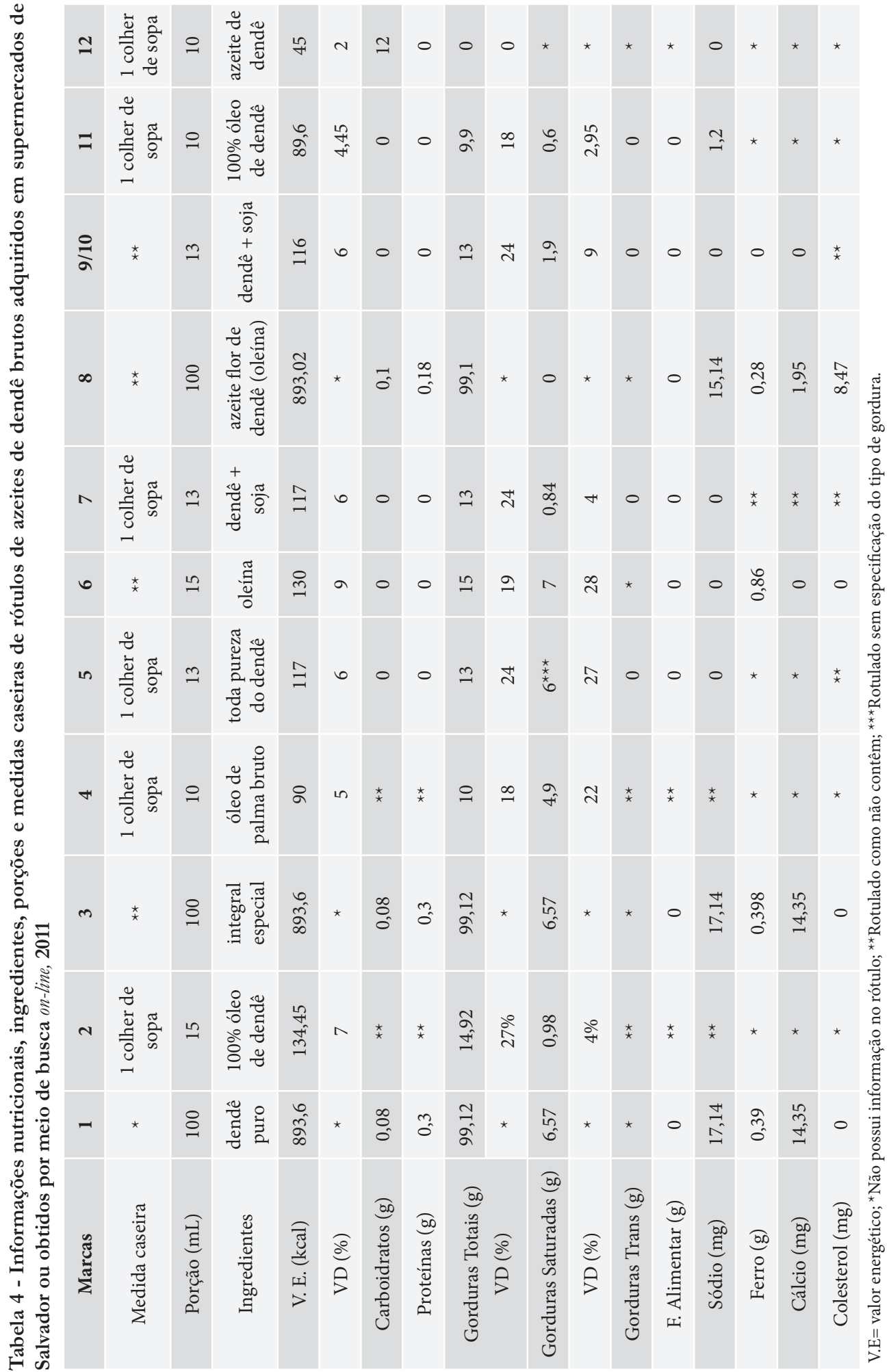


na composição, e a margarina "sabor milho" não apresentava, na lista de ingredientes, componentes de milho. Não se justificam as rotulagens das margarinas "sabor manteiga", "vitaminada", dado que "aroma idêntico à manteiga" e vitaminas A, D e E são ingredientes adicionados em margarinas de fabricação semelhante.

Chama-se a atenção para o fato de que 11,8\% dos produtos denominavam-se "manteiga + margarina" ou alimentos "à base de manteiga e margarina", mas tal nomenclatura não está prevista na legislação vigente ${ }^{11}$. Tais produtos eram de um mesmo fabricante e continham a alegação de " $50 \%$ menos colesterol".

Margarina cremosa e margarina extra cremosa não apresentaram diferenças estatísticas significativas quando comparadas ao produto "margarina + manteiga" para nenhum dos itens avaliados ( $\mathrm{p} \geq 0,05)$; o resultado é similar para margarina cremosa versus extra. A comparação entre os demais produtos, incluindo os cremes vegetais, demonstraram diferenças significantes $(\mathrm{p} \leq 0,05)$ quanto a kcal, gorduras totais e saturadas (Tabela 1).

Observou-se que 32,5\% dos produtos apresentaram frases não previstas no regulamento técnico ${ }^{12}$ tais como: "cozinha melhor", "qualidade de vida", "Vital energia e vitalidade", "faz tudo com sabor", "extrassaborosa”, entre outras, que podem induzir o consumidor a erros. Foi verificada conformidade em 100\% das margarinas e cremes vegetais em relação à declaração dos teores de fibras, proteínas, carboidratos (todos ausentes), com base em uma dieta de $2000 \mathrm{kcal}$. Todos atenderam aos valores declarados de medidas caseiras e porções, salientando-se que um dos cremes vegetais recomendava, em seu site, que seriam necessários $20 \mathrm{~g} /$ porção para atingir suas propriedades ditas hipocolesterolêmicas e, no rótulo comercializado no supermercado, apresentava $10 \mathrm{~g} /$ porção, o que é a metade do valor orientado no site para se obter os benefícios hipocolesterolêmicos do produto; esse quantitativo de porção foi informado no rótulo porque é o limite permitido em legislação. Os cálculos dos Valores Diários (VD\%) não estavam em conformidade em apenas duas marcas analisadas, referindo-se estes a gordura saturada e trans.

Seguindo uma tendência mundial de reduzir nos alimentos o teor de ácidos graxos trans, os quais são substâncias prejudiciais à saúde ${ }^{16,17}$, observou-se que $20,6 \%$ dos produtos eram hidrogenados e 79,4\% interesterificados. Verificou-se que, no rótulo de uma margarina interesterificada, foram declarados os valores de 1,3 g de trans/10 g e, antagonicamente, $0 \%$ deste item na tampa da embalagem. A RDC n⿳360 preconiza para a alegação zero trans o valor máximo de $0,2 \mathrm{~g}$ por porção. Dos produtos hidrogenados, dois foram cremes vegetais $(0,0$ a $0,3 \mathrm{~g}$ de gordura trans/ porção); dois, "manteiga + margarina" (1,0 a 1,3 g de gordura trans/porção); e um, margarina extra cremosa (1,3 g de gordura trans/porção), os quais são valores elevados, considerando-se que a IDR de gordura trans recomendada pela OMS é de 2 g. O Ministério da Saúde (MS) e a Associação Brasileira das Indústrias de Alimentos (ABIA) assinaram um acordo entre 2007 e 2010 com o intuito de limitar a presença de gordura trans nos alimentos processados, a 5\% e $2 \%$ do total de gorduras em óleos e margarinas ${ }^{18}$, respectivamente. Sendo assim, a porção de $10 \mathrm{~g}$ de margarina deveria conter no máximo $0,1 \mathrm{~g}$ de trans, valor extrapolado em 11,8\% nas margarinas e cremes vegetais analisados. 
MANTEIGA

A Portaria n 146, do Ministério da Agricultura, Pecuária e Abastecimento (MAPA) ${ }^{9}$, define como manteiga o o produto é classificado em extra e de primeira qualidade, conforme análise sensorial gorduroso obtido exclusivamente pela bateção e malaxagem, com ou sem modificação biológica de creme pasteurizado derivado exclusivamente do leite de vaca, por processos tecnologicamente adequados. A matéria gorda da manteiga deverá estar composta exclusivamente de gordura láctea; o produto é classificado em extra e de primeira qualidade, conforme análise sensorial. Já a Resolução $\mathrm{n}^{\circ} 4^{13}$, institui a Manteiga Comum, como creme obtido a partir do desnate do soro, regulamentada apenas no mercado brasileiro.

A análise dos ingredientes demonstrou que $61,3 \%$ das manteigas não especificavam a pasteurização do creme de leite. A Portaria $n^{\circ} 146^{9}$ e a Resolução $n^{\circ} 4^{13}$ determinam que às manteigas sejam adicionados de $2 \%$ a $3 \%$ de cloreto de sódio, respectivamente, ou seja, os produtos deveriam apresentar como limites máximos de 80 a $120 \mathrm{mg}$ de sódio por $100 \mathrm{~g}$ do produto. Verificou-se que os valores são díspares quanto à adição deste ingrediente, sendo que $20 \%$ das amostras ultrapassaram os valores estipulados, chegando ao dobro das diferenças (Tabela 2). De fato, o sódio foi o único nutriente estatisticamente diferente $(\mathrm{p} \leq 0,05)$ entre todos os produtos avaliados, exceto quando comparadas a manteiga comum e sem classificação $(p=0,09)$ e de primeira qualidade com manteiga extra com sal. Ressaltar que estas últimas não diferiram entre si em nenhum dos itens analisados ( $\mathrm{p} \geq 0,05)$ (Tabela 2).

Verificou-se ausência de informações sobre proteínas (6,7\%), carboidratos $(6,7 \%)$ e gorduras trans $(11,1 \%)$, medidas caseiras $(6,7 \%)$ e porções $(4,4 \%)$, nos rótulos das manteigas analisadas. Além disso, $6,8 \%$ destes produtos apresentaram informações incorretas quanto ao conteúdo de proteínas $(4,2 \mathrm{~g})$, carboidratos $(9,3 \mathrm{~g})$ e gorduras totais $(25 \mathrm{~g})$ e $29 \%$ em relação à porção estabelecida ${ }^{4}$.

Ainda que não seja de caráter obrigatório, 6,7\% das manteigas declararam teor de colesterol de 35; 45; e $196 \mathrm{mg}$ em $14 \mathrm{~g}$ do produto. Neste caso, apenas o primeiro valor se aproxima do encontrado em manteigas (em torno de $200 \mathrm{mg} / 100 \mathrm{~g}$ do produto) ${ }^{19}$. Considerando-se que uma porção de manteiga de $10 \mathrm{~g}$ aporta cerca de $12 \%$ das necessidades diárias de colesterol e os efeitos negativos desta substância sobre a gênese das doenças cardiovasculares ${ }^{20}$, seria recomendável a impressão obrigatória desta informação nos rótulos.

\section{REQUEIJÃO}

O requeijão é um produto tipicamente brasileiro classificado como: requeijão de corte; o culinário de bisnaga; o de balde; e o requeijão cremoso; mas existem outras variedades, como o requeijão do norte ${ }^{21}$. De acordo com a Portaria n ${ }^{0} 359$, do $\mathrm{MAPA}^{10}$, o requeijão possui como ingrediente básico o leite. Ainda segundo a citada portaria, o produto só pode ser reconhecido dessa forma se for

[...] obtido pela fusão da massa coalhada, cozida ou não, dessorada e lavada, obtida por coagulação ácida e/ou enzimática do leite, opcionalmente adicionada de creme de leite e/ou manteiga e/ ou gordura anidra de leite ou butter oil, sendo designado: "Requeijão", "Requeijão cremoso" ou "Requeijão de manteiga".

Todas as marcas analisadas demonstraram conformidade em relação aos ingredientes e seus $\operatorname{aditivos}^{10} ; 14,6 \%$ e $19,5 \%$ dos produtos não declararam corretamente a porção e a medida caseira, 
respectivamente ${ }^{4}$. Em relação à lista de nutrientes, apenas três amostras não atenderam à RDC $\mathrm{n}^{\circ} 360^{5}$, pois não foi declarada a informação de teor de gordura saturada em dois produtos e de gordura trans em um produto. Verificaram-se incorreções nos cálculos de VD (\%) em 34,1\% das amostras estudadas. Os produtos light apresentaram o teor médio de gorduras totais atendendo à regulamentação que estabelece uma redução de $25 \%$ destas em tais produtos ${ }^{12}$ (Tabela 3).

$\mathrm{Na}$ formulação de "requeijão com" é utilizado amido e/ou gordura vegetal, podendo conter também concentrado proteico de soro, fibras e outros ingredientes ${ }^{20}$. Constatou-se que, das 5 marcas declaradas com gordura e amido, uma não continha carboidratos. Ressalta-se a ausência de informações nutricionais de tais substâncias nas tabelas de composição de alimentos ${ }^{19,22,23}$.

Dos nutrientes analisados, o conteúdo de gorduras totais e saturadas estão de acordo com pesquisa realizada pelo Instituto Nacional de Metrologia, Qualidade e Tecnologia ${ }^{24}$.

A comparação estatística entre os requeijões demonstrou diferenças significantes para todos os itens analisados, exceto para o cálcio $(\mathrm{p}=0,524)$ e sódio $(\mathrm{p}=0,110)$ (Tabela 3). Destaca-se que $41,5 \%$ das amostras declararam teores de cálcio com VD de até $170 \mathrm{mg}$ por porção (17\% de VD) (Tabela 3), representando, uma boa fonte deste nutriente, sendo importante a sua declaração nos rótulos.

Quanto ao sódio, não se observaram diferenças significantes entre os requeijões tradicional e light $(\mathrm{p}=0,110)$, ainda que os resultados tenham sido bastante heterogêneos, com uma variação de VD \% de 2\% a 17\% (Tabela 3). De acordo com dados da Coordenação Geral de Alimentação e Nutrição do Ministério da Saúde, o consumo diário de sal no Brasil atualmente é de 12 gramas, enquanto o recomendado pela Organização Mundial de Saúde (OMS) é de apenas 5 gramas $^{1}$. Diante deste panorama, o Ministério da Saúde lançou a campanha, juntamente com as associações que representam os produtores de alimentos processados, "Menos sal sua saúde agradece", que estabelece uma redução no consumo excessivo de sal em 16 categorias de alimentos, incluindo os requeijões e as margarinas ${ }^{25}$.

\section{AzEITE DE DENDÊ}

Observou-se que 75,0\% das marcas analisadas não atendem à RDC n ${ }^{\circ} 359^{4}$, no que se refere aos valores declarados de porção $(13 \mathrm{~mL}), 50,0 \%$ não informam a medida caseira correspondente, e $58,3 \%$ não estão conformes em relação à declaração de VD \% com base em uma dieta de $2000 \mathrm{kcal}$. Os ingredientes não estão listados em 50\% das amostras, conforme a RDC n⿳360 subtendendo-se o seu conteúdo apenas com o nome do produto. Também se observou ausência de declaração do teor e VD \% das gorduras saturadas, fibra alimentar e de gorduras trans em $8 \%$, $3 \%, 8,3 \%$ e $41,7 \%$ dos rótulos, respectivamente (Tabela 4). Chama-se a atenção à declaração de $12 \mathrm{~g}$ de carboidratos em uma das amostras, informação totalmente equivocada para qualquer tipo de óleo ${ }^{26}$. Vale ressaltar a declaração do teor e VD\% de sódio, cálcio, ferro e de colesterol nas amostras analisadas (Tabela 4), o que não condiz com a composição de azeite de dendê bruto ${ }^{26}$. Cabe salientar que $41,5 \%$ das amostras não declararam VD \% para nutriente algum aqui listado.

Verificou-se que uma das amostras analisadas declarou valor calórico de $450 \mathrm{kcal} / 100 \mathrm{~g}$, o que difere substancialmente dos descritos nas três principais tabelas de composição de alimentos utilizadas no Brasil ${ }^{19,22,23}$. 


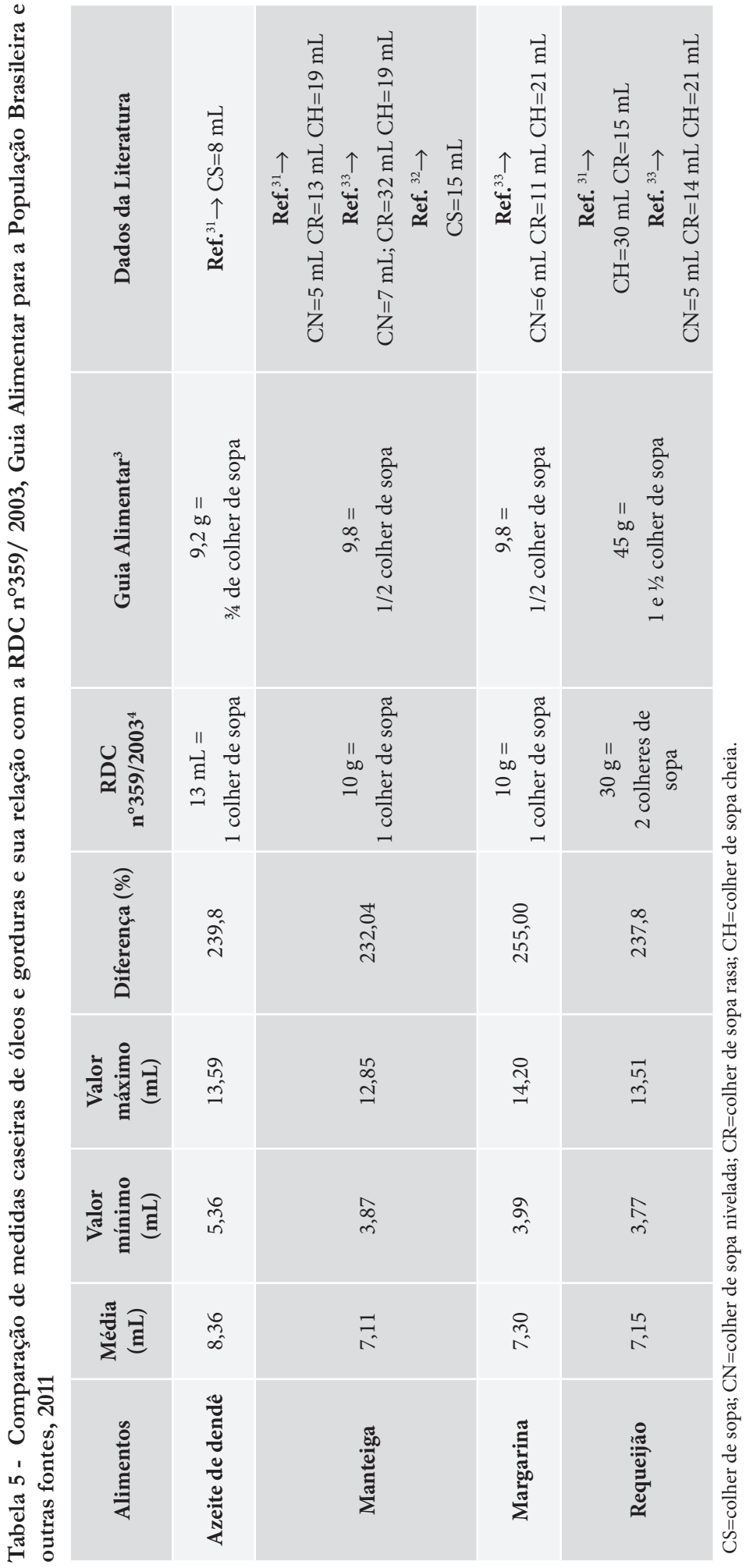


Em contrapartida, as maiores discrepâncias foram verificadas nos teores declarados de gorduras saturadas, em 41,6\% dos produtos, principalmente quanto aos valores relacionados ao óleo de palma bruto. Tal informação está incorreta (Tabela 4), dado que o óleo de palma bruto (Elaeis guineensis), comercializado no Brasil como azeite de dendê, contém cerca de 50,0 \% de ácidos graxos saturados, $40,0 \%$ de monoinsaturados e $10,0 \%$ de polinsaturados ${ }^{26}$.

No caso de azeite de dendê e óleo de soja, as composições esperadas dependem da proporção de cada um na mistura. O óleo de soja sem adição de dendê apresenta entre 15\% a 16\% de gordura saturada ${ }^{19,27,28}$. Tavares et al. ${ }^{29}$, ao analisarem a composição de ácidos graxos em misturas de azeite de dendê e óleo de soja, encontraram o máximo de 44,94\% e o mínimo de 17,46\% de gorduras saturadas; nesta última, a formulação continha maior teor de óleo de soja. A Tabela 5 demonstra que a quantidade de gordura saturada por $100 \mathrm{~g}$ seria de $14,62 \%$ e 6,46\% na mistura de dendê e soja. Portanto, no primeiro caso, a composição de gorduras saturadas poderia corresponder à soja e, no segundo, se aproximaria ao óleo de canola $(7,9 \%)^{19}$.

\section{MEDIDAS CASEIRAS E PORÇÕES}

A ANVISA, por meio da RDC $\mathrm{n}^{\circ} 359^{4}$, estabeleceu, para efeito de declaração na rotulagem nutricional, a medida caseira e sua relação com a porção correspondente em gramas ou mililitros, sendo previsto para uma colher de sopa $10 \mathrm{~cm}^{3}$ ou $\mathrm{mL}$. Esta resolução é conflitante quando comparada ao "Guia Alimentar para a População Brasileira ${ }^{3}$, uma vez que tais medidas e porções para um mesmo alimento diferem das sugeridas neste último documento (Tabela 5).

Ao contrário de outros países como os Estados Unidos da América, cuja regulamentação de padrões de utensílios de medidas permite uma variação máxima de $5 \%{ }^{6}$, o Brasil não dispõe de legislação específica sobre o tema, o que dificulta a exatidão das medidas. Esta situação está refletida nos dados apresentados, cujas diferenças percentuais da capacidade volumétrica das colheres de sopa chegam a $200 \%$, coadunando com as pesquisas de Victora \& Coelho $^{30}$ e Botelho ${ }^{6}$, as quais mensuraram a capacidade volumétrica de colheres de sopa e observaram uma variação de volume entre 8,73 a 17,46 e 9,00 a $17,5 \mathrm{~mL}$, respectivamente.

Ressalta-se que apenas $6,7 \% ; 6,7 \% ; 5,0 \%$ e 4,4 \% das colheres apresentaram capacidade volumétrica para margarina e cremes vegetais, manteiga, requeijão e azeite de dendê, respectivamente, dentro do preconizado pela legislação $(10 \mathrm{~mL} \mathrm{ou} \mathrm{g})^{4}$, e a maioria apresentou valor inferior (de 75,6\% a 86,7\%) ao recomendado. Os valores médios dos pesos dos alimentos estudados situaram-se entre $7 \mathrm{~g}$ e $8 \mathrm{~g}$ (Tabela 5) e a literatura relata capacidade volumétrica para colheres de sopa cheias entre $19 \mathrm{~g}$ e $30 \mathrm{~g}^{31-33}$ diferindo significativamente do recomendado. Vale salientar que a diferença entre as capacidades volumétricas ultrapassou 200\% (Tabela 5).

Considerando-se estas prerrogativas, as porções estabelecidas necessitam ser revistas, principalmente no que se refere ao seu porcionamento quanto às colheres de sopa; niveladas; rasas; ou cheias. Usualmente a população utiliza a terminologia "ponta da faca" para descrever a quantidade desses produtos untados em pães, torradas e biscoitos. Tal forma de mensuração poderia/deveria ser agregada à rotulagem de tais produtos, oferecendo um protocolo de operacionalização de medidas comumente utilizado e reconhecido pelo consumidor. Em alguns países, os produtos comercializados em tabletes possuem uma prévia divisão/marcação em gramatura definida na própria embalagem ${ }^{34}$, o que também poderia ser uma conduta a ser adotada 
pelo governo e indústrias de alimentos brasileiros, a fim de complementar a legislação e garantir o porcionamento correto ao consumidor.

\section{CONCLUSÃO}

Ocorreram discrepâncias importantes na informação nutricional e nos ingredientes em todos os produtos estudados, sendo que a amostra que apresentou maior número de itens não conformes foi a do azeite de dendê, para qualquer das marcas analisadas. A legislação de margarinas e cremes vegetais deveria ser revista, uma vez que a atual permite aos fabricantes uma ampla utilização de termos para produtos similares e outros não previstos em leis.

É notória a necessidade de uma legislação específica para a padronização de utensílios culinários domésticos, como os talheres, haja vista que as diferenças encontradas na capacidade volumétrica das colheres refletem diretamente a inadequação da porção estabelecida, e, portanto, afeta diretamente a ingestão de nutrientes recomendados.

As legislações de rotulagem no Brasil foram um marco de conquista do consumidor, sendo um dos fatores de influência na determinação dos hábitos alimentares da população. No entanto, apesar de quase uma década desde a sua publicação, nota-se uma série de não conformidades nos rótulos dos alimentos analisados e a necessidade de uma fiscalização ostensiva para tornar essas legislações mais eficazes às necessidades dos consumidores.

\section{REFERÊNCIAS/REFERENCES}

1. World Health Organization - WHO. Global Strategy on Diet, Physical Activity and Health [internet]. [cited 2011 Dec 20]. Available from: http://www. who.int/dietphysicalactivity/strategy/en/.

2. Barreto SM, Pinheiro ARO, Sichieri R, Monteiro CA, Batista M Fo, Schimidt MI, et al. Análise da estratégia global para alimentação, atividade física e saúde, da Organização Mundial da Saúde. Epidemiol Serv Saúde. 2005;14(1):41-68. http:/ / dx.doi.org/10.5123/S1679-49742005000100005

3. Brasil. Ministério da Saúde. Guia alimentar para a população brasileira: Promovendo a alimentação saudável. Secretaria de Atenção à Saúde, Coordenação-Geral da Política de Alimentação e Nutrição. Brasília: Ministério da Saúde, 2005. 236 p. (Série A. Normas e Manuais Técnicos).

4. Agência Nacional de Vigilância Sanitária - Anvisa Resolução RDC nº.359, de 23 de dezembro de 2003. Aprova o regulamento técnico de porções de alimentos embalados para fins de rotulagem nutricional. Diário Oficial da República Federativa do Brasil; 2003.

5. Brasil. Ministério da Saúde. Agência Nacional de Vigilância Sanitária - Anvisa Resolução RDC n 360, de 23 de dezembro de 2003. Aprova o regulamento técnico sobre rotulagem nutricional de alimentos embalados. Diário Oficial da República Federativa do Brasil; 2003.

6. Botelho RA. Características dos utensílios alimentícios no Brasil e seu impacto na construção de tabelas de pesos e medidas caseiras. Nutr Brasil. 2007;6(2):105-110

7. Câmara MCC, Marinho CLC, Guilam CM, Braga AMCB. A produção acadêmica sobre a rotulagem de alimentos no Brasil. Rev Panam Salud Publica. 2008; 23(1):52-58. http://dx.doi.org/10.1590/ S1020-49892008000100007

8. Lobanco MC, Vedovato MG, Cano CB, Bastos MHD. Fidedignidade de rótulos de alimentos comercializados no município de São Paulo, SP. Rev 
Saúde Pública. 2009;43(3):499-505. http://dx.doi. org/10.1590/S0034-89102009005000020

9. Brasil. Portaria $n^{\circ} 146$ de 1996. Regulamento Técnico para Fixação de Identidade e Qualidade de Manteiga. Diário Oficial da República Federativa do Brasil; 1996 [cited 2011 Dec 21]. Available from: http://extranet.agricultura. gov.br/sislegisconsulta/consultarLegislacao. do operacao $=$ visualizar\&id $=1218$.

10. Brasil. Ministério da Agricultura e do Abastecimento. Secretaria de Defesa Agropecuária. Departamento de Inspeção de Produtos de Origem Animal. Portaria $n^{\circ} 359$. Regulamento técnico para fixação de identidade e qualidade do requeijão cremoso ou requesón. Diário Oficial da República Federativa do Brasil; 1997a.

11. Brasil. Ministério da Agricultura. Divisão de Inspeção de Produtos de Origem Animal (DIPOA). Portaria $\mathrm{n}^{\circ}$ 372, de 04 de Setembro de 1997. Regulamento técnico para fixação de identidade e qualidade de margarina. Diário Oficial da República Federativa do Brasil; 1997b.

12. Brasil. Ministério da Saúde. Secretaria de Vigilância Sanitária. Resolução da Diretoria Colegiada - RDC $\mathrm{n}^{\circ} 54$, de 12 de novembro de 2012a. Regulamento técnico referente à informação nutricional complementar. Diário Oficial da República Federativa do Brasil; 2012. [cited 2014 Feb 18]. Available from: http://portal.anvisa.gov.br/ wps/wcm/connect/630a98804d7065b981f1e 1c116238c3b/Resolucao+RDC+n.+54_2012. pdf?MOD=AJPERES

13. Brasil. Resolução $\mathrm{n}^{\circ} 4$ de 28 de junho de 2000 . Institui a Manteiga Comum. Diário Oficial da República Federativa do Brasil; 2000. [cited 2011 Dec 21]. Available from: http://extranet.agricultura. gov.br/sislegisconsulta/consultarLegislacao. do?operacao $=$ visualizar\&id $=3284$.

14. Agência Nacional de Vigilância Sanitária. Resolução RDC n 270, 2005. Aprova o Regulamento Técnico para óleos vegetais, gorduras vegetais e creme vegetal. Diário Oficial da República Federativa do Brasil; 2005 [cited 2011 Dec 21]. Available from: http://portal.anvisa.gov.br/wps/wcm/connect/82
d8d2804a9b68849647d64600696f00/RDC_n_270. pdf?MOD=AJPERES.

15. Philippi ST. Nutrição e Técnica Dietética. 5. ed. São Paulo: Manole; 2003.

16. Cavendish TA, Lemos PB, Yokota RT, Vasconcelos TF, Coêlho PF, Buzzi M, et al. Composição de ácidos graxos de margarinas à base de gordura hidrogenada ou interesterificada. Ciênc Tecnol Aliment. 2010;30(1):138-142. http://dx.doi. org/10.1590/S0101-20612010005000018

17. Costa AGV, Bressan J, Sabarense CM. Ácidos Graxos Trans: Alimentos e Efeitos na Saúde. ALAN. 2006;56(1):12-21. Available from:http:// www.scielo.org.ve/scielo.php?

18. Brasil. Ministério da Saúde. Renova acordo com indústria para melhorar alimentação dos brasileiros [Internet]. 2012b [cited $2012 \mathrm{Mar} 6$ ]. Available from: http://portal.saude.gov.br/portal/arquivos/pdf/ nota65_pdf.

19. Universidade Estadual de Campinas - UNICAMP. Tabela brasileira de composição de alimentos - TACO. 4. ed. Campinas: UNICAMP/ NEPA; 2001 [cited 2011 Nov 27]. Available from: http://www.unicamp.br/nepa/taco/tabela. php?ativo=tabela.

20. Houston DK, Ding J, Lee JS, Garcia M, Kanaya AM, Tylavsky FA, Newman AB, Visser M, Kritchevsky SB. Dietary fat and cholesterol and risk of cardiovascular disease in older adults: The Health ABC Study. Nutr Met Card Dis. 2011;21(6):430437. PMid:20338738 PMCid:PMC2911502. http:// dx.doi.org/10.1016/j.numecd.2009.11.007

21. Van Dender AGF. Requeijão cremoso e outros queijos fundidos: tecnologia de fabricação, controle do processo e aspectos de mercado. São Paulo: Comunicações e Editora; 2006.

22. Franco G. Tabela de composição química dos alimentos. 9. ed. São Paulo: Atheneu; 1992.

23. 23 Instituto Brasileiro de Geografia e Estatística - IBGE. Tabela de composição de alimentos. 4. ed. Rio de Janeiro: IBGE, Estudo Nacional da Despesa Familiar - ENDEF; 1996. 
24. Instituto Nacional de Metrologia, Qualidade e Tecnologia - INMETRO [internet]. Requeijão e especialidade láctea à base de requeijão. 2006 [cited 2011 Nov 25]. Available from: http://www.inmetro. gov.br/consumidor/produtos/requeijao.asp

25. Coordenação Geral de Alimentação e Nutrição [internet]. Construção da agenda de reformulação de alimentos processados com as indústrias de alimentação. [cited 2011 Dec 06]. Available from: http://nutricao.saude.gov.br/sodio_reformulacao. php.

26. Codex Alimentarius. Codex standard for named vegetable oils, Codex Stan 210 (Amended 20032005). Review 2011.

27. Barros HD, Vianni R. Estudo da influência da área específica nas alterações do óleo de soja utilizado em frituras descontínuas com batata inglesa. Higiene Alimentar. 2006;20:76-82.

28. Jorge N, Malacrida CR. Alterações do óleo de soja e da mistura azeite de dendê - óleo de soja em frituras descontínuas de batatas chips. Brazilian Journal of Food Technol. 2003;6(2):245-249.

29. Tavares M, Barbério JC, Badolato ESG, Maio FD, Carvalho JB. Identificação e quantificação de adulterantes do óleo de dendê por meio de cromatografia em fase gasosa. Rev Adolfo Lutz. 1989;49(2):145-150.

30. Victora CG, Coelho VH. Volume of household measures: a matter of concern. J Trop Pediatr. 1989;29(3):133-134. http://dx.doi.org/10.1093/ tropej/29.3.133

31. Pinheiro ABV, Lacerda EMA, Benzecry EH, Gomes MCS, Costa VM. Tabela para Avaliação de Consumo Alimentar em Medidas Caseiras. 5. ed. Rio de Janeiro: Atheneu, 1996.

32. Paiotti J. Arte e Técnica na Cozinha. Glossários multilíngue, métodos e receitas. 5. ed. São Paulo: Livraria Varela Editora; 2004.

33. Moreira MA. Medidas Caseiras no Preparo de Alimentos. 2. ed. Goiânia: Cultura e Qualidade; 2002.

34. Wikipedia. Size and shape of butter packaging [internet] [cited 2014 Feb 03]. Available from: http://en.wikipedia.org/wiki/Butter.

Recebido para publicação em 03/05/13. Aprovado em 12/02/14. 